\title{
Phase-I monitoring of standard deviations in multistage linear profiles
}

\author{
Mahdiyeh Kalaei $^{1} \cdot$ Paria Soleimani $^{2} \cdot$ Seyed Taghi Akhavan Niaki $^{3}$. \\ Karim Atashgar ${ }^{1}$
}

Received: 20 September 2016/Accepted: 8 June 2017/Published online: 28 June 2017

(c) The Author(s) 2017. This article is an open access publication

\begin{abstract}
In most modern manufacturing systems, products are often the output of some multistage processes. In these processes, the stages are dependent on each other, where the output quality of each stage depends also on the output quality of the previous stages. This property is called the cascade property. Although there are many studies in multistage process monitoring, there are fewer works on profile monitoring in multistage processes, especially on the variability monitoring of a multistage profile in Phase-I for which no research is found in the literature. In this paper, a new methodology is proposed to monitor the standard deviation involved in a simple linear profile designed in Phase I to monitor multistage processes with the cascade property. To this aim, an autoregressive correlation model between the stages is considered first. Then, the effect of the cascade property on the performances of three types of $T^{2}$ control charts in Phase I with shifts in standard deviation is investigated. As we show that this effect is significant, a $U$ statistic is next used to remove
\end{abstract}

Paria Soleimani

p_soleimani@azad.ac.ir

Mahdiyeh Kalaei

m.kalaei@yahoo.com

Seyed Taghi Akhavan Niaki

niaki@sharif.edu

Karim Atashgar

atashgar@iust.ac.ir

1 Industrial Engineering Department, Malek Ashtar University of Technology, 15875-1774 Tehran, Iran

2 Industrial Engineering Department, South Tehran Branch, Islamic Azad University, Tehran, Iran

3 Industrial Engineering Department, Sharif University of Technology, 11155-9414 Tehran, Iran the cascade effect, based on which the investigated control charts are modified. Simulation studies reveal good performances of the modified control charts.

Keywords Multistage processes - Cascade property . Profile monitoring · Phase I

\section{Introduction and literature review}

In many production processes, the quality of a product is better monitored by a linear relationship between the quality characteristic and one or more explanatory variables. This relationship is referred to as a profile. Mestek et al. (1994), Stover and Brill (1998), Kang and Albin (2000), Kim et al. (2003), and Mahmoud and Woodall (2004) are just a few researchers who worked on profile monitoring. In addition, Niaki et al. (2007), Saghaei et al. (2009), Zhang et al. (2009), Zhu and Lin (2010), Noorossana et al. (2010), Amiri et al. (2011) and Narvand et al. (2013) are some researchers who worked on Phase-II profile monitoring. Besides, Du et al. (2015a, b) investigated the cascade effects in multistage processes.

As the focus in this paper is Phase-I monitoring, some recent and relevant works are reviewed here. Mahmoud et al. (2007) introduced a change-point method for linear profiles in Phase I. Kazemzadeh et al. (2008) applied three methods, namely a change point, $F$ statistics and $T^{2}$, to monitor polynomial profiles in Phase I. Other studies in Phase I include Jensen et al. (2008), Noorossana et al. (2009), Zou et al. (2007) and Khedmati and Niaki (2015a).

In some applications, it is necessary to monitor multistage processes. For example, there are three dependent stages in a production process of Nylon Yarn factory schematically depicted in Fig. 1. A textile industry is 


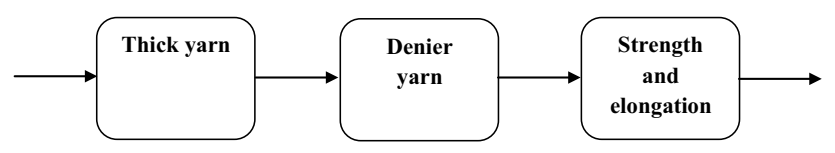

Fig. 1 A three-stage process for Nylon yarn production

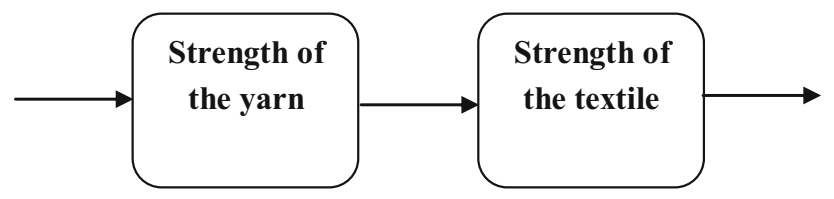

Fig. 2 A two-stage process of weaving

another three-stage process involving spinning, weaving and finishing. In this type of industry, there are two successive stages in the weaving process. In the first stage, the strength of the yarn is increased and checked. In the next stage, the strength of the textile in different parts is considered as a profile that is affected by the strength of the yarn (see Fig. 2).

In multistage processes, the stages are generally correlated and the use of profile monitoring without paying attention to the relation between the stages causes monitoring errors. Phase-II monitoring of linear profiles in multistage processes has been investigated by authors including Esmaeeli and Sadeghieh (2013) and Eghbali Ghahyazi et al. (2014). Soleimani et al. (2009) studied the presence of within-profile autocorrelation in simple profile monitoring. Moreover, Atashgar et al. (2014) investigated the Phase-I monitoring problem of multistage processes using the $T^{2}$ scheme of Stover and Brill (1998), the $T^{2}$ control chart of Kang and Albin (2000), and the $T^{2}$ chart of Williams et al. (2007). They studied the effect of the cascade property on the test power factor by changing the slope and the intercept parameters. Moreover, Atashgar et al. (2015) investigated the effect of the cascade property when the $T^{2}$ chart of Williams et al. (2007) was used for Phase-I monitoring. In this study, the shifts were imposed on the slope and intercept of a simple linear profile. In another work, Khedmati and Niaki (2015b) considered Phase-I profile monitoring of general linear profiles in multistage processes. In addition, Khedmati and Niaki (2016) focused on profile monitoring of multistage processes using a EWMA control chart. Recently, Kalaei et al. (2016) proposed a methodology to first remove the effect of the cascade property in multistage processes. Then, they developed a control chart for Phase-I monitoring of simple linear profiles.

The review of the relevant studies indicates that Phase-I variability monitoring of profiles in multistage processes involving the cascade property has not been investigated yet. As the performance of a profile monitoring scheme is highly dependent on the standard deviation of the error term in a regression model being used, this paper focuses on Phase-I monitoring of the standard deviation of a simple linear regression profile in a multistage process with cascade property. To this aim, based on Du and Zhang (2016) who discussed the joint monitoring of input and output of systems using autoregressive disturbances of arbitrary orders, a two-stage process is first modeled in this paper by an autoregressive model of order one, i.e., AR(1). Then, the shifts are applied on the standard deviation of a simple linear profile. Similar to Atashgar et al. (2014), three methods in Phase-I profile monitoring including the $T^{2}$ control chart proposed by Stover and Brill (1998), the $T^{2}$ scheme of Kang and Albin (2000), and the $T^{2}$ chart of Williams et al. (2007) are employed to investigate the effect of the cascade property. Eventually a new control chart based on the $U$ statistic is proposed for Phase-I monitoring of the standard deviation in multistage processes. This statistic has been shown to be very effective to remove the effect of the cascade property (Kalaei et al. 2016).

The structure of the remainder of this paper is as follows. In Sect. 2, the problem is defined, the assumptions are made and the two-stage process is modeled. The investigation of the effect of the cascade property on the test power when the standard deviation is changed is performed in Sect. 3. The new control chart based on the $U$ statistic is proposed in Sect. 4. Section 5 is about the performance evaluation of the new methodology. Section 6 as a final part is dedicated to the remarkable conclusions.

\section{Problem definition, assumptions, and modeling}

As mentioned above, quality characteristics of a multistage process in any stage are affected not only by the performance of that stage but also by the ones of the previous stages, implying the so-called cascade property. Ignoring this property and assuming independent stages leads into large Type-I errors.

To take into account the cascade property, a multistage process can be expressed by the following equations:

$y_{i k 1}=A_{0}+A_{1} x_{i}+\varepsilon_{i k 1} \quad i=1,2, \ldots, n$

$y_{i k s}=\emptyset y_{i k(s-1)}+\alpha_{1 s}+\alpha_{2 s} x_{i}+\varepsilon_{i k s}, \quad s=2,3, \ldots, S$,

where for the $k$ th sample, $\left(x_{i}, y_{i k 1}\right)$ and $\left(x_{i}, y_{i k s}\right)$ are the $i$ th observation for the first and the sth stages, respectively. In addition, $A_{0}$ and $A_{1}$ are the intercept and the slope of the first simple linear profile, respectively, and $x_{i}$ is an independent variable with constant values such as $0,0.2,0.4$, 0.6, 0.8, 1, 1.2, 1.4, 1.6 and 1.8 (Mahmoud and Woodall 2004). Moreover, $\alpha_{1 s}$ and $\alpha_{2 s}$ are the special effects of stage 
$s$ on the intercept and slope, respectively, $\emptyset$ is the autocorrelation parameter, and $\varepsilon_{i k 1}$ and $\varepsilon_{i k s}$ are the random error terms with normal distribution having mean 0 and variances $\sigma_{1}^{2}$ and $\sigma_{s}^{2}$ for the first and the $s$ th stages, respectively. For simplicity $\sigma_{1}^{2}=\sigma_{s}^{2}=\sigma^{2}$ is considered.

As a Phase-I profile monitoring of multistage processes is considered in this paper, it is necessary to estimate the process parameters. Due to the independency of the first stage, $A_{0}$ and $A_{1}$. are estimated, respectively, by $a_{0 k 1}$ and $a_{1 k 1}$ using the least squares method. Then, the estimates of the intercept and the slope in the second stage, i.e., $a_{0 k 2}$ and $a_{1 k 2}$, are obtained based on Eqs. (1) and (2) as

$a_{0 k 2}=\emptyset a_{0 k 1}+\alpha_{1}+\varepsilon_{k 1}^{\prime \prime}$

$a_{1 k 2}=\emptyset a_{1 k 1}+\alpha_{2}+\varepsilon_{k 1}^{\prime}$,

where,

$\varepsilon_{k 1}^{\prime \prime}=\frac{\sum_{i=1}^{n} \varepsilon_{i k 2}}{n}-\bar{x} \varepsilon_{1}^{\prime}$

and

$\varepsilon_{k 1}^{\prime}=\frac{\sum_{i=1}^{n}\left(x_{i}-\bar{x}\right) \varepsilon_{i k 2}}{s_{x x}}$

have a normal distribution with mean zero and variance $\sigma^{2}\left(\frac{1}{n}+\frac{\vec{x}^{2}}{s_{x x}}\right)$ and $\frac{\sigma^{2}}{s_{x x}}$, respectively. This leads into the mean and the variance of the estimator of the intercept in the second stage as

$E\left(a_{0 k 2}\right)=\emptyset A_{0}+\alpha_{1}$

$\operatorname{Var}\left(a_{0 k 2}\right)=\left(1+\emptyset^{2}\right) \cdot \sigma^{2}\left(\frac{1}{n}+\frac{\bar{x}^{2}}{s_{x x}}\right)$.

Moreover, the mean and the variance of the estimator of the slope in the second stage are

$E\left(a_{1 k 2}\right)=\emptyset A_{1}+\alpha_{2}$

$\operatorname{Var}\left(a_{1 k 1}\right)=\left(1+\emptyset^{2}\right) \frac{\sigma^{2}}{s_{x x}}$.

Furthermore, the co-variance estimator is

$\operatorname{Cov}\left(a_{0 k 2}, a_{1 k 2}\right)=-\left(1+\emptyset^{2}\right) \frac{\bar{x} \sigma^{2}}{s_{x x}}$.

Interested readers are referred to Eghbali Ghahyazi et al. (2014) for more details on Eqs. (7)-(11).

\section{Evaluating the cascade effect on the test power of the control scheme}

The effect of the cascade property on the out-of-control signal detection of a monitoring chart is investigated in this section by assessing the test power when the standard deviation 
Table 2 The values of the test power for the change in the standard deviation from $\sigma$ to $\lambda \sigma$

\begin{tabular}{|c|c|c|c|c|c|c|c|c|c|c|}
\hline & \\
\hline & 1.2 & 1.4 & 1.6 & 1.8 & 2.0 & 2.2 & 2.4 & 2.6 & 2.8 & 3.0 \\
\hline \multicolumn{11}{|c|}{$T^{2}$ of Kang and Albin } \\
\hline$\emptyset=0$ & 0.1709 & 0.1734 & 0.1776 & 0.1789 & 0.1786 & 0.1776 & 0.1771 & 0.1780 & 0.1784 & 0.1793 \\
\hline$\emptyset=0.1$ & 0.1717 & 0.1752 & 0.1817 & 0.1773 & 0.1785 & 0.1800 & 0.1774 & 0.1817 & 0.1825 & 0.1839 \\
\hline$\emptyset=0.3$ & 0.1764 & 0.1778 & 0.1831 & 0.1779 & 0.1819 & 0.1825 & 0.1868 & 0.1951 & 0.1978 & 0.2081 \\
\hline$\emptyset=0.5$ & 0.1723 & 0.1820 & 0.1808 & 0.1865 & 0.2028 & 0.2128 & 0.2405 & 0.2468 & 0.2625 & 0.2812 \\
\hline$\emptyset=0.7$ & 0.1802 & 0.1886 & 0.1948 & 0.2110 & 0.2240 & 0.2568 & 0.2787 & 0.3132 & 0.3488 & 0.3741 \\
\hline$\emptyset=0.9$ & 0.1800 & 0.1874 & 0.2102 & 0.2322 & 0.2600 & 0.2927 & 0.3366 & 0.3792 & 0.4077 & 0.4446 \\
\hline \multicolumn{11}{|c|}{$T^{2}$ of Stover and Brill } \\
\hline$\emptyset=0$ & 0.1825 & 0.1823 & 0.1817 & 0.1848 & 0.1855 & 0.1952 & 0.1961 & 0.2027 & 0.2036 & 0.2045 \\
\hline$\emptyset=0.1$ & 0.1971 & 0.1947 & 0.1887 & 0.1951 & 0.1852 & 0.1876 & 0.1898 & 0.1939 & 0.1991 & 0.2050 \\
\hline$\emptyset=0.3$ & 0.1972 & 0.1925 & 0.1931 & 0.1942 & 0.1955 & 0.2006 & 0.2016 & 0.2045 & 0.2088 & 0.2152 \\
\hline$\emptyset=0.5$ & 0.1933 & 0.1914 & 0.1979 & 0.2037 & 0.2191 & 0.2283 & 0.2343 & 0.2598 & 0.2797 & 0.3006 \\
\hline$\emptyset=0.7$ & 0.1900 & 0.1997 & 0.2079 & 0.2200 & 0.2431 & 0.2715 & 0.3015 & 0.3341 & 0.3804 & 0.4137 \\
\hline$\emptyset=0.9$ & 0.1910 & 0.2016 & 0.2164 & 0.2562 & 0.2798 & 0.3174 & 0.3652 & 0.3990 & 0.4530 & 0.5002 \\
\hline \multicolumn{11}{|c|}{$T^{2}$ of Williams } \\
\hline$\emptyset=0$ & 0.2482 & 0.2495 & 0.2502 & 0.2518 & 0.2521 & 0.2508 & 0.2523 & 0.2553 & 0.2590 & 0.2626 \\
\hline$\emptyset=0.1$ & 0.2615 & 0.2568 & 0.2602 & 0.2605 & 0.2637 & 0.2645 & 0.2663 & 0.2697 & 0.2755 & 0.2897 \\
\hline$\emptyset=0.3$ & 0.2665 & 0.2636 & 0.2679 & 0.2718 & 0.2725 & 0.2775 & 0.2804 & 0.2916 & 0.2974 & 0.3020 \\
\hline$\emptyset=0.5$ & 0.2604 & 0.2686 & 0.2750 & 0.2892 & 0.2952 & 0.3143 & 0.3281 & 0.3504 & 0.3744 & 0.3936 \\
\hline$\emptyset=0.7$ & 0.2661 & 0.2689 & 0.2857 & 0.2999 & 0.3292 & 0.3533 & 0.3806 & 0.4142 & 0.4476 & 0.4811 \\
\hline$\emptyset=0.9$ & 0.2667 & 0.2856 & 0.3162 & 0.3389 & 0.3678 & 0.3928 & 0.4347 & 0.4752 & 0.5192 & 0.5519 \\
\hline
\end{tabular}

parameter is changed. Three control schemes are considered here; the $T^{2}$ chart of Kang and Albin (2000), the $T^{2}$ scheme of Stover and Brill (1998), and the $T^{2}$ chart of Williams et al. (2007). Table 1 contains the statistics used in these methods.

Suppose the two regression models shown in Eqs. (12) and (13) have been defined for the first- and the secondstage profiles in a two-stage process, respectively.

$y_{i k 1}=3+2 x_{i}+\varepsilon_{i k 1}$

$y_{i k 2}=\left(3 \emptyset+\alpha_{1}\right)+\left(2 \emptyset+\alpha_{2}\right) x_{i}+\emptyset \varepsilon_{i k 1}+\varepsilon_{i k 2}$.

Let $x_{i}=\left[\begin{array}{llllllllll}0 & 0.2 & 0.4 & 0.6 & 0.8 & 1 & 1.2 & 1.4 & 1.6 & 1.8\end{array}\right]$, $\left(3 \emptyset+\alpha_{1}\right)=2$, and $\left(2 \emptyset+\alpha_{2}\right)=1$ (Eghbali Ghahyazi et al. 2012), where $\varepsilon_{i k 1}$ and $\varepsilon_{i k 2}$ follow the normal distribution with mean zero and variance 1. Then, Table 2 and Figs. 3, 4 and 5 show the detection probability of the out-of-control signal for the shifts in the standard deviation of the firststage profile from $\sigma$ to $\sigma \lambda$ based on various values of the autocorrelation parameter. The estimates are obtained using 10,000 simulation runs, where $\alpha$ (type one error) is assumed 0.01 , and $\lambda=1.2,1.4,1.6,1.8,2.0,2.2,2.4,2.6$, 2.8,3.0. In this analysis, the standard deviation of the process is shifted in the first stage and the test power is obtained for the second stage.

The figures clearly show that the test power has an increasing trend in all methods and for all autocorrelation

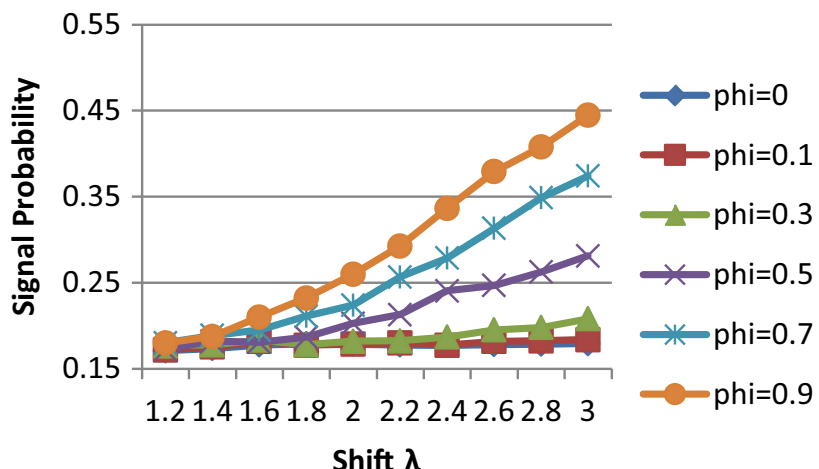

Fig. 3 Test power of $T_{2}$ of Kang and Albin (2000) chart under the shifts in the standard deviation of Stage 1 from $\sigma$ to $\sigma \lambda$

coefficients. In other words, when the shift size in the standard deviation of the first stage increases, the power of detecting an out-of-control signal in the second stage increases for all levels of $\emptyset$. This increase occurs faster for bigger values of $\emptyset$, implying that for a given shift in the standard deviation of the first stage, the cascade property that is enhanced when $\emptyset$ increases leads into an undesired higher Stage-2 detection power. The results in Table 1 as well as in Figs. 3, 4 and 5 also show that the $T^{2}$ of Knag 


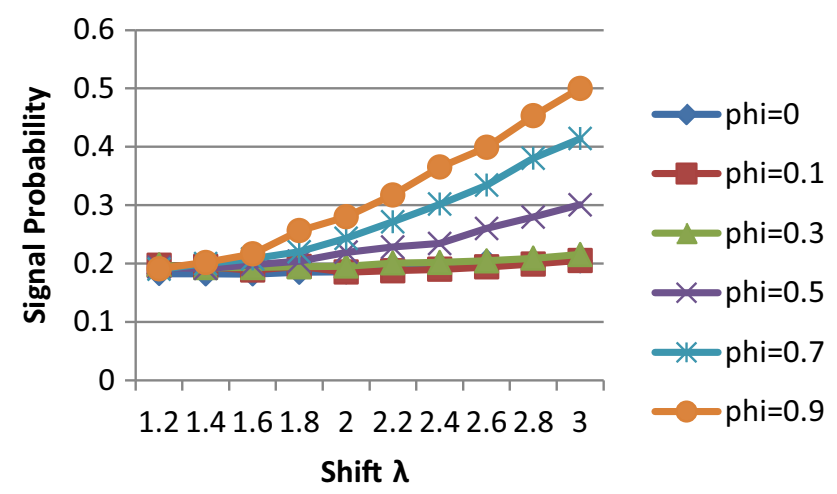

Fig. 4 Test power of $T_{2}$ of Stover \& Brill (1998) scheme under the shifts in the standard deviation of Stage 1 from $\sigma$ to $\sigma \lambda$

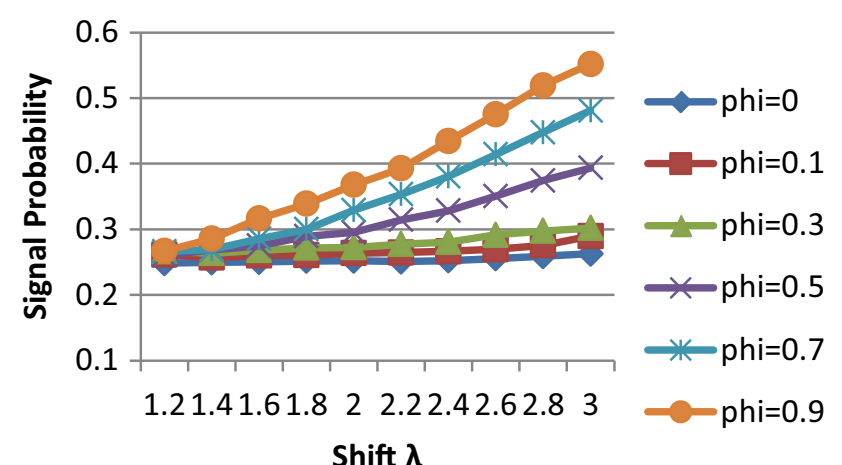

Fig. 5 Test power of $T 2$ of Williams et al. (2007) chart under the shifts in the standard deviation of Stage 1 from $\sigma$ to $\sigma \lambda$

and Albin (2000) has the best performance, as the cascade property has the least effect on its test power.

\section{The $U$ method to remove the cascade property}

As mentioned, the cascade property of a multistage process affects the test power of a control scheme to rise undesirably. Thus, removing this property may lead into a test power that is a good indicative of the performance of a stage, no matter what happened in its previous stage.
To eliminate or to reduce the cascade property, a control plan is modified using the $U$ statistic proposed by Hauck et al. (1999). For the $j$ th profile in the first stage of a multistage process, in this plan we have

$U_{j 1}=A_{1}$.

In addition for stage $s$ we have:

$U_{j s}=A_{s}-\sum_{s(s-1)} \sum_{(s-1)(s-1)}^{-1} A_{(s-1)}$,

where $A_{\mathrm{s}-1}$ and $A_{\mathrm{s}}$ are the vector estimators of the intercept and slope parameters in stage $s-1$ and $s$, respectively, $\sum_{s(s-1)}$ is the covariance matrix of the parameters between stage $s$ and stage $s-1$, and $\sum_{(s-1)(s-1)}$ is variance-covariance matrix of the estimators at stage $s-1$ (see Hauck et al. 1999 for more details).

The mean vector and the covariance matrix for the $U$ transformation in the first and sth stage are obtained, respectively, as follows:

$\mu_{U_{j 1}}=\mu_{A_{1}}$

$\Sigma_{U_{j 1}}=\Sigma_{A_{1}}=\Sigma_{11}$

$\mu_{U_{j s}}=\mu_{A_{s}}-\sum_{s(s-1)} \sum_{(s-1)(s-1)}^{-1} \mu_{A_{(s-1)}}$

$\Sigma_{U_{j s}}=\sum_{s s}-\sum_{s(s-1)} \sum_{(s-1)(s-1)}^{-1} \sum_{(s-1) s}$.

Then, the $U$-transformation when employed for the $T^{2}$ of Kang and Albin (2000) scheme for example, the statistic becomes:

$T_{U_{j s}}^{2}=\left(U_{j s}-\mu_{U_{s}}\right) \sum_{U_{s}}^{-1}\left(U_{j s}-\mu_{U_{s}}\right)^{\mathrm{T}}$.

The upper control limit for this statistic in each stage is $\chi_{\alpha, 2}^{2}$. (Mahmoud and Woodall 2004). Although similar statistics can be obtained for the other two schemes as well, since the aim is to investigate the performance of the $U$ statistic, they are not discussed in this paper.
Table 3 Test power of the $U$ method under the shifts in standard deviation of Stage 1 from $\sigma$ to $\sigma \lambda$

\begin{tabular}{lllllllllll}
\hline$\lambda$ & 1.2 & 1.4 & 1.6 & 1.8 & 2 & 2.2 & 2.4 & 2.6 & 2.8 & 3 \\
\hline$\emptyset=0$ & 0.1353 & 0.1335 & 0.1327 & 0.1323 & 0.1318 & 0.1324 & 0.1333 & 0.1321 & 0.1312 & 0.1402 \\
$\emptyset=0.1$ & 0.1275 & 0.1326 & 0.1313 & 0.1351 & 0.1282 & 0.133 & 0.1327 & 0.1292 & 0.1327 & 0.1374 \\
$\emptyset=0.3$ & 0.1319 & 0.1356 & 0.1314 & 0.1265 & 0.1344 & 0.129 & 0.1325 & 0.1335 & 0.1336 & 0.1296 \\
$\emptyset=0.5$ & 0.1323 & 0.1304 & 0.1238 & 0.1274 & 0.1338 & 0.1293 & 0.1296 & 0.1308 & 0.1352 & 0.133 \\
$\emptyset=0.7$ & 0.1315 & 0.1312 & 0.1325 & 0.1353 & 0.1312 & 0.1312 & 0.1342 & 0.127 & 0.129 & 0.1353 \\
$\emptyset=0.9$ & 0.1348 & 0.127 & 0.127 & 0.1274 & 0.1293 & 0.1341 & 0.1296 & 0.1349 & 0.129 & 0.1355 \\
\hline
\end{tabular}



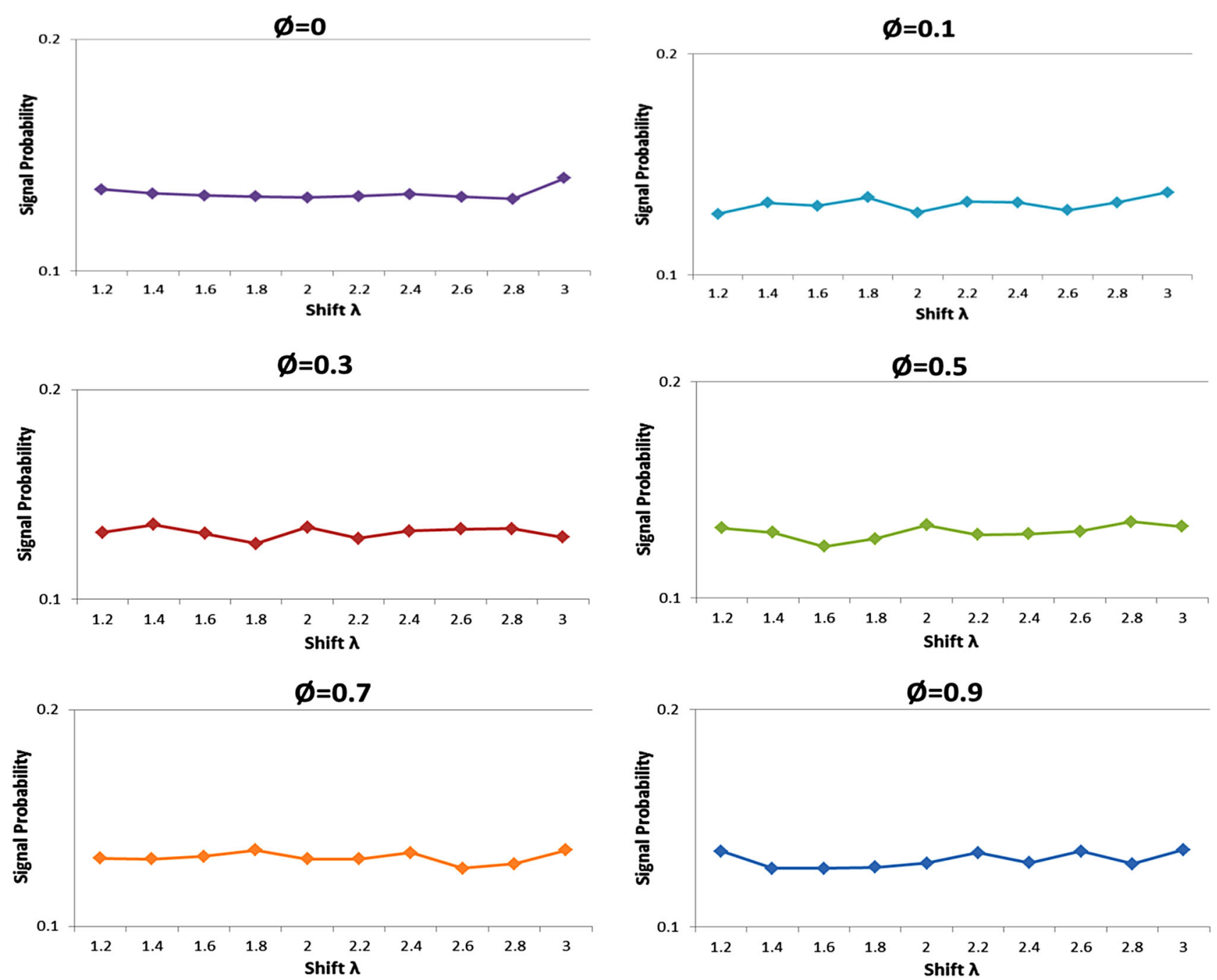

Fig. 6 Test power of the $U$ method under the shifts in standard deviation of Stage 1 from $\sigma$ to $\sigma \lambda$

\section{Performance evaluation of $\boldsymbol{U}$ method}

In this section, the performance of the $U$ method for PhaseI monitoring of simple linear profiles when used for twostage process is investigated. Kalaei et al. (2016) investigated similar performances when slope and intercept parameters were subject to changes. In this paper, however, the performance of the $\mathrm{U}$ method is investigated for the shift in the standard deviation in two cases:

1. Different shifts in the standard deviation involved in the first stage and

2. Different shifts in the standard deviation involved in the first and in the second stages of the process, simultaneously.

Table 3 and Fig. 6 show the probability of detecting an out-of-control situation in the second stage of the process for a shift on the standard deviation of the first stage from $\sigma$ to $\sigma \lambda$ when the $\mathrm{U}$ method is employed (Case 1 above). The estimates are obtained based on 10,000 simulation runs, assuming $\alpha=0.01$ and $\lambda=1.2,1.4,1.6,1.8,2.0,2.2$, 2.4, 2.6, 2.8, and 3.0.

As seen in Fig. 6, when the $U$ method is applied the test power values are not influenced by the value of $\emptyset$ and are distributed randomly. It means that the performance of the $\mathrm{U}$ method for different $\emptyset$ values is unchanged. On the other hand, Fig. 6 shows that the values of the test power are between 0.12 and 0.14 for different $\emptyset$ values. It indicates that the proposed approach has the robust property to monitor a simple linear profile in a multistage process.

A similar investigation is performed on the performance of the $U$ methods in Case 2, when simultaneous shifts are imposed on the standard deviations of both stages. Table 4 contains the probabilities of detecting an out-of-control condition when the standard deviations of the first and the second stage of the process are changed from $\sigma_{1}$ to $\lambda_{1} \sigma_{1}$ 
Table 4 Test powers of the $U$ method under the shifts in standard deviations of the first and the second stages of the process from $\sigma_{1}$. to $\lambda_{1} \sigma_{1}$ and from $\sigma_{2}$ to $\lambda_{2} \sigma_{2}$, respectively

\begin{tabular}{|c|c|c|c|c|c|c|c|c|c|c|c|}
\hline & $\lambda_{1}$ & 1.2 & 1.4 & 1.6 & 1.8 & 2 & 2.2 & 2.4 & 2.6 & 2.8 & 3 \\
\hline \multirow[t]{5}{*}{$\lambda_{1}=1.2$} & $\emptyset=0.1$ & 0.4515 & 0.7809 & 0.9386 & 0.9848 & 0.9967 & 0.9997 & 1 & 1 & 1 & 1 \\
\hline & $\emptyset=0.3$ & 0.4526 & 0.7799 & 0.9365 & 0.9845 & 0.9975 & 0.9996 & 0.9999 & 1 & 1 & 1 \\
\hline & $\emptyset=0.5$ & 0.4448 & 0.7666 & 0.9336 & 0.9843 & 0.9964 & 0.9994 & 1 & 1 & 1 & 1 \\
\hline & $\emptyset=0.7$ & 0.4522 & 0.7739 & 0.9348 & 0.9865 & 0.9969 & 0.9996 & 0.9997 & 0.9999 & 1 & 1 \\
\hline & $\emptyset=0.9$ & 0.4485 & 0.7795 & 0.9393 & 0.9822 & 0.9966 & 0.9997 & 0.9999 & 0.9999 & 1 & 1 \\
\hline \multirow[t]{5}{*}{$\lambda_{1}=1.4$} & $\emptyset=0.1$ & 0.4566 & 0.7717 & 0.9386 & 0.9851 & 0.9984 & 0.9999 & 0.9999 & 1 & 1 & 1 \\
\hline & $\emptyset=0.3$ & 0.4505 & 0.7810 & 0.9369 & 0.9860 & 0.9968 & 0.9995 & 1 & 1 & 1 & 1 \\
\hline & $\emptyset=0.5$ & 0.4579 & 0.7785 & 0.9346 & 0.9876 & 0.9975 & 0.9996 & 0.9998 & 1 & 1 & 1 \\
\hline & $\emptyset=0.7$ & 0.4566 & 0.7755 & 0.9355 & 0.9859 & 0.9971 & 0.9993 & 1 & 1 & 1 & 1 \\
\hline & $\emptyset=0.9$ & 0.4533 & 0.7786 & 0.9381 & 0.9865 & 0.9966 & 0.9993 & 0.9999 & 1 & 1 & 1 \\
\hline \multirow[t]{5}{*}{$\lambda_{1}=1.6$} & $\emptyset=0.1$ & 0.4387 & 0.7704 & 0.9367 & 0.9868 & 0.9964 & 0.9994 & 1 & 1 & 1 & 1 \\
\hline & $\emptyset=0.3$ & 0.4606 & 0.7783 & 0.9338 & 0.9856 & 0.9969 & 0.9994 & 1 & 1 & 1 & 1 \\
\hline & $\emptyset=0.5$ & 0.4474 & 0.7781 & 0.9359 & 0.9877 & 0.9967 & 0.9996 & 0.9999 & 1 & 1 & 1 \\
\hline & $\emptyset=0.7$ & 0.4475 & 0.7782 & 0.9353 & 0.985 & 0.9983 & 0.9992 & 1 & 1 & 1 & 1 \\
\hline & $\emptyset=0.9$ & 0.4499 & 0.7702 & 0.9344 & 0.984 & 0.9973 & 0.9997 & 0.9999 & 1 & 1 & 1 \\
\hline \multirow[t]{5}{*}{$\lambda_{1}=1.8$} & $\emptyset=0.1$ & 0.4566 & 0.7716 & 0.9326 & 0.9838 & 0.9975 & 0.9997 & 1 & 1 & 1 & 1 \\
\hline & $\emptyset=0.3$ & 0.4589 & 0.7779 & 0.9385 & 0.9844 & 0.9978 & 0.9994 & 0.9999 & 1 & 1 & 1 \\
\hline & $\emptyset=0.5$ & 0.4512 & 0.7823 & 0.9500 & 0.9850 & 0.9977 & 0.9997 & 0.9999 & 1 & 1 & 1 \\
\hline & $\emptyset=0.7$ & 0.4593 & 0.7806 & 0.9404 & 0.9866 & 0.9978 & 0.9997 & 0.9999 & 1 & 1 & 1 \\
\hline & $\emptyset=0.9$ & 0.4496 & 0.7747 & 0.9368 & 0.9975 & 0.9998 & 0.9999 & 1 & 1 & 1 & 1 \\
\hline \multirow[t]{5}{*}{$\lambda_{1}=2$} & $\emptyset=0.1$ & 0.4570 & 0.7711 & 0.9328 & 0.986 & 0.9976 & 0.9996 & 0.9999 & 0.9998 & 1 & 1 \\
\hline & $\emptyset=0.3$ & 0.4491 & 0.7727 & 0.9381 & 0.9848 & 0.9971 & 0.9999 & 0.9999 & 1 & 1 & 1 \\
\hline & $\emptyset=0.5$ & 0.4471 & 0.7657 & 0.9323 & 0.9851 & 0.9970 & 0.9993 & 1 & 1 & 1 & 1 \\
\hline & $\emptyset=0.7$ & 0.4544 & 0.7775 & 0.9352 & 0.9854 & 0.9971 & 0.9995 & 0.9999 & 1 & 1 & 1 \\
\hline & $\emptyset=0.9$ & 0.4538 & 0.7721 & 0.9347 & 0.9868 & 0.9964 & 0.9997 & 0.9997 & 1 & 1 & 1 \\
\hline \multirow[t]{5}{*}{$\lambda_{1}=2.2$} & $\emptyset=0.1$ & 0.4526 & 0.7803 & 0.9377 & 0.9836 & 0.9977 & 0.9992 & 0.9999 & 1 & 1 & 1 \\
\hline & $\emptyset=0.3$ & 0.4573 & 0.7781 & 0.9395 & 0.9833 & 0.9966 & 0.9994 & 0.9999 & 1 & 1 & 1 \\
\hline & $\emptyset=0.5$ & 0.4568 & 0.7748 & 0.9357 & 0.9849 & 0.9975 & 0.9996 & 1 & 1 & 1 & 1 \\
\hline & $\emptyset=0.7$ & 0.4598 & 0.7754 & 0.9304 & 0.9861 & 0.9975 & 0.9997 & 1 & 1 & 1 & 1 \\
\hline & $\emptyset=0.9$ & 0.4493 & 0.7811 & 0.9375 & 0.9868 & 0.9964 & 0.9996 & 0.9999 & 1 & 1 & 1 \\
\hline \multirow[t]{5}{*}{$\lambda_{1}=2.4$} & $\emptyset=0.1$ & 0.4600 & 0.7706 & 0.9363 & 0.9869 & 0.9977 & 0.9994 & 0.9998 & 0.9999 & 1 & 1 \\
\hline & $\emptyset=0.3$ & 0.4515 & 0.7794 & 0.9339 & 0.9849 & 0.9973 & 0.9993 & 0.9999 & 1 & 1 & 1 \\
\hline & $\emptyset=0.5$ & 0.4495 & 0.7789 & 0.9324 & 0.9817 & 0.9974 & 0.9997 & 0.9998 & 1 & 1 & 1 \\
\hline & $\emptyset=0.7$ & 0.4533 & 0.7731 & 0.9347 & 0.9852 & 0.9972 & 0.9991 & 1 & 1 & 1 & 1 \\
\hline & $\emptyset=0.9$ & 0.4610 & 0.7704 & 0.9408 & 0.9870 & 0.9976 & 0.9992 & 1 & 1 & 1 & 1 \\
\hline \multirow[t]{5}{*}{$\lambda_{1}=2.6$} & $\emptyset=0.1$ & 0.4611 & 0.778 & 0.9304 & 0.9816 & 0.9967 & 0.999 & 0.9998 & 0.9999 & 1 & 1 \\
\hline & $\emptyset=0.3$ & 0.4527 & 0.7745 & 0.9341 & 0.9852 & 0.9967 & 0.9994 & 0.9996 & 0.9999 & 1 & 1 \\
\hline & $\emptyset=0.5$ & 0.4528 & 0.7721 & 0.9320 & 0.9869 & 0.9978 & 0.9994 & 0.9998 & 1 & 1 & 1 \\
\hline & $\emptyset=0.7$ & 0.4553 & 0.7865 & 0.9343 & 0.9851 & 0.9973 & 0.9996 & 0.9999 & 1 & 1 & 1 \\
\hline & $\emptyset=0.9$ & 0.4410 & 0.7778 & 0.9381 & 0.9849 & 0.9973 & 0.9996 & 1 & 1 & 1 & 1 \\
\hline \multirow[t]{5}{*}{$\lambda_{1}=2.8$} & $\emptyset=0.1$ & 0.4536 & 0.7785 & 0.9351 & 0.9863 & 0.9973 & 0.9994 & 0.9999 & 1 & 1 & 1 \\
\hline & $\emptyset=0.3$ & 0.4523 & 0.7764 & 0.9378 & 0.9852 & 0.9967 & 0.9993 & 0.9999 & 1 & 1 & 1 \\
\hline & $\emptyset=0.5$ & 0.4521 & 0.7722 & 0.9392 & 0.9848 & 0.9963 & 0.9991 & 0.9997 & 1 & 1 & 1 \\
\hline & $\emptyset=0.7$ & 0.4442 & 0.7695 & 0.9321 & 0.9854 & 0.9968 & 0.9998 & 0.9999 & 1 & 1 & 1 \\
\hline & $\emptyset=0.9$ & 0.4579 & 0.7783 & 0.9329 & 0.9853 & 0.9955 & 0.9996 & 0.9997 & 1 & 1 & 1 \\
\hline
\end{tabular}


Table 4 continued

\begin{tabular}{|c|c|c|c|c|c|c|c|c|c|c|c|}
\hline & $\lambda_{1}$ & 1.2 & 1.4 & 1.6 & 1.8 & 2 & 2.2 & 2.4 & 2.6 & 2.8 & 3 \\
\hline \multirow[t]{5}{*}{$\lambda_{1}=3$} & $\emptyset=0.1$ & 0.4518 & 0.7837 & 0.9396 & 0.9846 & 0.9976 & 0.9993 & 0.9996 & 0.9999 & 1 & 1 \\
\hline & $\emptyset=0.3$ & 0.4529 & 0.7807 & 0.9327 & 0.9847 & 0.9977 & 0.9991 & 1 & 1 & 1 & 1 \\
\hline & $\emptyset=0.5$ & 0.4630 & 0.7730 & 0.9336 & 0.9844 & 0.9972 & 0.9996 & 0.9999 & 1 & 1 & 1 \\
\hline & $\emptyset=0.7$ & 0.4496 & 0.7811 & 0.9354 & 0.9875 & 0.9971 & 0.9995 & 0.9998 & 1 & 1 & 1 \\
\hline & $\emptyset=0.9$ & 0.4467 & 0.7695 & 0.9302 & 0.9855 & 0.9980 & 0.9993 & 0.9999 & 1 & 1 & 1 \\
\hline
\end{tabular}
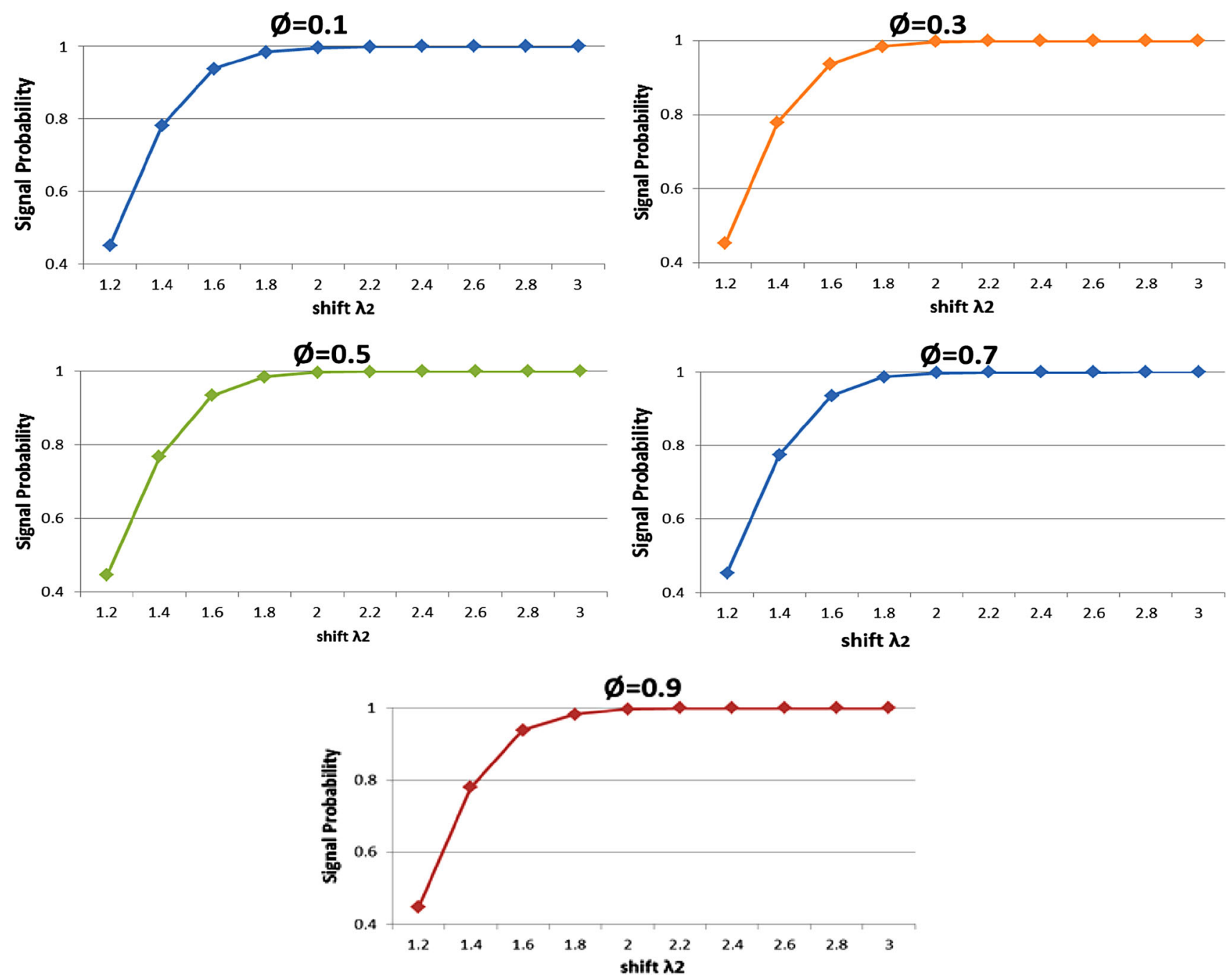

Fig. 7 Test powers of the $U$ method under the shifts in standard deviations of Stage 1 from $\sigma_{1}$ to $\lambda_{1} \sigma_{1}\left(\lambda_{1}=1.2\right)$ and Stage 2 from $\sigma_{2}$ to $\lambda_{2} \sigma_{2}$

and from $\sigma_{2}$ to $\lambda_{2} \sigma_{2}$, respectively, where $\lambda_{1}=\lambda_{1}=$ 1.2, 1.4, 1.6, 1.8, 2.0, 2.2, 2.4, 2.6, 2.8, and 3.0. Again, the estimates are obtained based on 10,000 simulation runs and $\alpha=0.01$. Figure 7 is a plot of the test power results when the shift value of first stage is $1.2 \sigma_{1}$. The other plots for the other values of $\lambda_{1}$ show a similar pattern.

As seen in Fig. 7, when simultaneous shifts of the standard deviations are imposed in both stages, the values of the test power are increased significantly between 0.4 and 1 (compared to the test powers in Case 1). This is due to the fact that the shifts are also considered in the second stage and the test powers are calculated in that stage as well. In this case, as the magnitude of the standard deviation shifts in the second stage of the process increases, the $U$ statistic is able to detect an out-of-control condition with a higher probability as desired. 


\section{Conclusion}

The cascade property involved in multistage processes has a significant effect on the performances of the control charts designed to monitor these processes. In this paper, this effect was first investigated for three control schemes proposed for Phase-I linear profile monitoring, namely $T^{2}$ of Kang and Albin (2000), $T^{2}$ of Stover and Brill (1998) and $T^{2}$ of Williams et al. (2007). Then, to remove or reduce the cascade property, a method using the $U$ transformation was developed for Phase-I monitoring of linear profiles in multistage processes. Two cases of the shifts in the standard deviations of a two-stage process were considered. The first case involved changes in the standard deviation of the first stage and the second case involved the changes in the standard deviations of both the first and the second stages, simultaneously. Next, the test powers of the developed method in detecting an out-of-control condition of the second stage of the process were investigated. Simulation studies showed a proper performance of the proposed method in removing the cascade property.

The use of a soft computing approach such as artificial neural network and decision tree is recommended for Phase-I monitoring of linear regression profiles in multistage processes.

Open Access This article is distributed under the terms of the Creative Commons Attribution 4.0 International License (http://crea tivecommons.org/licenses/by/4.0/), which permits unrestricted use, distribution, and reproduction in any medium, provided you give appropriate credit to the original author(s) and the source, provide a link to the Creative Commons license, and indicate if changes were made.

\section{References}

Amiri A, Eyvazian M, Zou C, Noorossana R (2011) A parameters reduction method for monitoring multiple linear regression profiles. Int J Adv Technol 58:621-629

Atashgar K, Niaki STA, Kalaei M, Soleimani P (2014) Evaluation of the shift identification power in multistage processes for Phase I of simple linear profile monitoring (In Farsi). J Qual Eng Manag $4: 1-14$

Atashgar K, Kalaei M, Soleimani P (2015). Analyzing power test for a two-stages processes Using T2 Williams procedure (in Farsi). International conference on management and industrial engineering

Du S, Zhang R (2016) Modelling and joint monitoring of input and output of systems with arbitrary order autoregressive disturbance. Int J Prod Res 54:1822-1838

Du S, Yao X, Huang D (2015a) Engineering model-based Bayesian monitoring of ramp-up phase of multistage manufacturing process. Int J Prod Res 53:4594-4613

Du S, Yao X, Huang D, Wang M (2015b) Three-dimensional variation propagation modeling for multistage turning process of rotary workpieces. Comput Ind Eng 82:41-53
Eghbali Ghahyazi M, Niaki STA, Soleimani P, Afroozan S (2012). Effect of cascade Property on the performance of simple linear profiles. Proceedings of the 2012 international conference on industrial engineering and operations management, Istanbul, Turkey, July 3-6

Eghbali Ghahyazi M, Niaki STA, Soleimani P (2014) On the monitoring of linear profiles in multistage processes. Qual Reliab Eng Int 30:1035-1047

Esmaeeli H, Sadegheih A (2013) Introduce and compare two approaches for monitoring a two-stage process by profile quality characteristic in the second stage. Res J Recent Sci 2:32-42

Hauck DJ, Runger GC, Montgomery DC (1999) Multivariate statistical process monitoring and diagnosis with grouped regression-adjusted variables. Commun Stat Simul Comput 28(2):309-328. doi:10.1080/03610919908813551

Jensen WA, Birch JB, Woodall WH (2008) Monitoring correlation within linear profiles using mixed models. J Qual Technol 40:167-183

Kalaei M, Atashgar K, Niaki STA, Soleimani P (2016) Phase-I monitoring of simple linear profiles in multistage processes with cascade property. Int J Adv Manuf Technol. doi:10.1007/ s00170-016-9691-0

Kang L, Albin SL (2000) On-line monitoring when the process yields a linear profile. J Qual Technol 32:418-426

Kazemzadeh RB, Noorossana R, Amiri A (2008) Phase I monitoring of polynomial profiles. Commun Stat Theory Methods 37:1671-1686

Khedmati M, Niaki STA (2015a) Identifying the time of a step change in $\mathrm{AR}(1)$ auto-correlated single linear profiles. J Ind Eng Int 11:473-484

Khedmati M, Niaki STA (2015b) Phase I monitoring of general linear profiles in multistage processes. Commun Stat Simul Comput. doi:10.1080/03610918.2015.1118507

Khedmati M, Niaki STA (2016) Monitoring simple linear profiles in multistage processes by a Max EWMA control chart. Comput Ind Eng 98:125-143

Kim K, Mahmoud MA, Woodall WH (2003) On the monitoring of linear profiles. J Qual Technol 35:317-328

Mahmoud AM, Woodall WH (2004) Phase I analysis of linear profiles with calibration applications. Technometrics 46:380-391

Mahmoud MA, Parker PA, Woodall WH, Hawkins DM (2007) A change point method for linear profile data. Qual Reliab Eng Int 23:247-268

Mestek O, Pavlik J, Suchanek M (1994) Multivariate control charts: control charts for calibration curves. Fresenius' J Anal Chem 350:344-351

Narvand A, Soleimani P, Raissi S (2013) Phase II monitoring of autocorrelated linear profiles using linear mixed model. J Ind Eng Int 9:12

Niaki STA, Abbasi B, Arkat J (2007) A generalized linear statistical model approach to monitor profiles. Int $\mathrm{J}$ Eng Trans $\mathrm{A}$ 20:233-242

Noorossana R, Eyvazian M, Amiri A, Mahmoud AM (2009) Statistical monitoring of multivariate multiple linear regression profiles in Phase I with calibration application. Qual Reliab Eng Int 26:291-303

Noorossana R, Eyvazian M, Vaghefi A (2010) Phase II monitoring of multivariate simple linear profiles. Comput Ind Eng 57:425-436

Saghaei A, Mehrjoo M, Amiri A (2009) A CUSUM-based method for monitoring simple linear profiles. Int $\mathbf{J}$ Adv Manuf Technol 45:1252

Soleimani P, Noorossana R, Amiri A (2009) Simple linear profile monitoring in the presence of within profile autocorrelation. Comput Ind Eng 57:1015-1021

Stover FS, Brill RV (1998) Statistical quality control applied to Ion chromatography calibrations. J Chromatogr A 804:37-43 
Williams JD, Woodall WH, Birch JB (2007) Statistical monitoring of nonlinear product and process quality profiles. Qual Reliab Eng Int 23:925-941

Zhang J, Li Z, Wang Z (2009) Control chart based on likelihood ratio for monitoring linear profiles. Comput Stat Data Anal 53:1440-1448
Zhu J, Lin OKJ (2010) Monitoring the slopes of linear profiles. Qual Eng 22:1-12

Zou C, Tsung F, Wang Z (2007) Monitoring general linear profiles using multivariate exponentially weighted moving average schemes. Technometrics 49:395-408 